\title{
Personalised Modelling on Integrated Clinical and EEG Spatio-Temporal Brain Data in the NeuCube Spiking Neural Network System
}

\author{
Maryam Gholami Doborjeh, Student member, IEEE, Nikola Kasabov, Fellow, IEEE \\ Knowledge Engineering and Discovery Research Institute, Auckland University of Technology, \\ Auckland 1010, New Zealand \\ mgholami@aut.ac.nz, nkasabov@,aut.ac.nz
}

\begin{abstract}
This paper introduces a novel personalised modelling framework and system for analysing Spatio-Temporal Brain Data (STBD) along with person clinical static data. For every individual, based on selected subset of similar to this individual clinical data, a subset of STBD is used for training a personalised Spiking Neural Network (PSNN) model using the recently proposed NeuCube SNN architecture. The proposed method is illustrated on a case study of personalised modelling using clinical and EEG data of two groups of subjects - drug addicts and addicts under medication.
\end{abstract}

The PSNN models help to achieve a better classification accuracy compared to global SNN models or when using traditional AI methods. A PSNN model visualisation enables discovery of new knowledge about individual persons and to distinguish complex STBD across subjects.

Keywords - personalised modelling; spiking neural networks; NeuCube; spatiotemporal data;EEG data; opiate addict; methadone maintenance treatment

\section{INTRODUCTION}

Personalised modelling is currently one of the main techniques for modelling and pattern discovery in the machine learning area. So far massive amount of patient static and dynamic datasets are available and need to be precisely analysed. Numerous personal events, such as personalised treatment for individual patient, can be obtained if we can model and learn the triggering patterns hidden deep in the patient data. Various techniques have been developed for recording brain dynamics and massive amount of Spatio-Temporal Brain Data (STBD), such as EEG, fMRI etc. [1], [2] have been accumulated. It becomes obvious that a unifying spatiotemporal computational approach is needed for proper analysis, understanding and utilisation of such complex STBD.

In this study, we introduce a personalised modelling approach which deals with person clinical static information as well as the person dynamic brain information as suggested in [9]. The model is based on SNN architecture. We demonstrate how SNN can be used to create efficient personalised modelling systems which reveal complex dynamic patterns that help understand individual person performance. The proposed system has been applied on EEG data case study for comparative analysis of personalised SNN models created for opiate addict patients versus those ones under methadone maintenance treatment. The models result in more accurate classification accuracy and better understanding of individual patient's response to methadone maintenance treatment. The results are also compared to global SNN models trained on the same EEG data, reported in [3].

Personalised modelling framework for static gene data analysis and biomedical applications was proposed by Kasabov in Integrated Model for Personalised Modelling (IMPM) [4]. In [9] and [20] this method was extended to the use of both static and dynamic, temporal personal data with the use of SNN and the method was demonstrated on stroke event prediction.

In the proposed method in this paper we introduce for the first time the integration of clinical, static data and EEG STBD using the NeuCube SNN architecture and the approach from [9] and [20].

The paper is organised as follows: section II explains the NeuCube SNN-based architecture; section III explains the personalised modelling approach based on the NeuCube and the used EEG data case study; section IV reports some experimental results.

\section{The NeuCube SNN-BASED Architecture}

Spiking Neural Networks (SNN) are computational models that consist of spiking neurons as processing units for modelling and learning data. In comparison with traditional neuronal networks, SNN can integrate both spatial and temporal information as locations of synapses and the time of their spiking activity respectively [5]-[10].

Several spiking neuronal models have been proposed so far. As one implementation, the popular Leaky-Integrate-and Fire Model (LIFM) is used here.

The paper uses a recently proposed SNN architecture NeuCube [10], [23] that refers also to elements of previous studies [11]-[17].

NeuCube consists of several functional modules [10], [18], and [23]:

- Input data encoding. 
- Input variables spatial mapping and unsupervised learning in a 3D brain-like SNNcube.

- Supervised learning in an output classification/regression module [8].

- Parameter optimisation.

- Visualisation and knowledge extraction.

Input data encoding: continuous streams of STBD are encoded into sequences of spikes using a Threshold-Based Representation method (TBR) as one implementation. Fig. 1 shows an example of single temporal variable encoding process. Then the encoded spike trains of the STBD variables will be entered into the SNNcube module for unsupervised training.

SNNcube has a 3D brain-like structure with recurrent connections. Initial neuronal connections are generated with the use of the "small world" connectivity rule. The spatial distance of two neurons is calculated to determine their initial connection weight. According to this rule, neurons within small area are more densely connected, and the weight of the connections are depended on the distance between the neurons

Each neuron in the SNNcube corresponds to a brain area according to a general brain template (such as Talairach [21], MNI, etc.). Each input neuron in the SNNcube has the same $(x, y, z)$ coordinates as the corresponding input data variable (EEG channel) in the used brain template. The input EEG spike trains are propagated through the SNNcube via the allocated input neurons and unsupervised learning is applied.

Unsupervised learning in a 3D SNNcube is performed using Spike-Timing Dependent Plasticity (STDP) learning rule [22] as one implementation. During the learning, efficacy of synapses is strengthened or weakened based on the timing of post-synaptic action potential in relation to the pre-synaptic spike. If pre-synaptic neuron $i$ spikes first and then postsynaptic neuron $j$ spikes, then the connection weight $W_{i j}$ between these two neurons $i$ and $j$ increases, otherwise it decreases.

Then a supervised learning in an output classification/regression module [8] is performed using dynamic evolving Spiking Neural Networks (deSNN) [8].

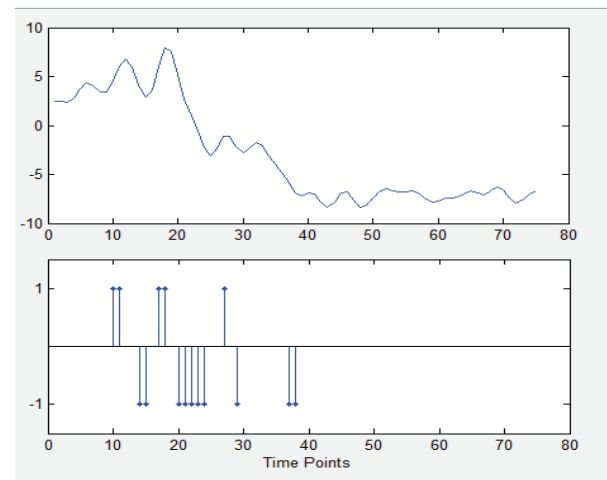

Fig. 1. An example of a STBD signal (top row) encoded into positive and negative spikes (below row) using a Threshold-Based representation method (TBR). The raw temporal data belongs to one EEG channel variable recorded over time. If the STBD signal increases more than a threshold, positive spikes are generated. If the STBD signal drops more than a threshold, negative spikes are generated
deSNN is computationally efficient model and emphasizes the importance of the first spike, which has been observed in biological systems. This is performed to train the output classifier neurons using class label information associated with the training samples. The output classifier is trained using the Rank-Order (RO) learning rule to initiate connection weights and a drift parameter to adjust them according to following spikes on the same synapse.

During the supervised training of an output deSNN, for every training sample (a labelled input spatio-temporal pattern), an individual output neuron $i$ is evolved and connected to all spiking neurons in the SNNcube. An output neuron is trained to recognize the spatio-temporal pattern of activity in the already trained SNNcube that is triggered when an individual spatiotemporal input pattern corresponding to this individual is propagated through the SNNcube.

\section{Personalised Modelling with a NeuCube SNN MODEL}

In this research, instead of building a global model and training it with STBD of the whole patient population, for every patient we will build a personalised SNN model to train it only on STBD of those patients that have similar static clinical factors. Patients with similar medical factors (drug types, long term or short term usage, methadone dos, etc.) may fall into a similar STBD pattern category due to similar medical effects on their brain functions.

We hypothesize that personalised modelling with SNN could be successfully used, if the PSNN models learn from the most informative STBD which are selected based on the similarity in the patients static data which is also the foundation of the method proposed in [9] and [20].

\section{A. Personalised SNN Models Trained on EEG STBD}

The proposed NeuCube personalised modelling for STBD is performed based on the following steps:

1. Select K-nearest neighbour vectors to a new individual vector $x 1$ from the global static data and form a cluster of similar samples with close proximity to vector $x 1$.

2. Select the STBD of the selected K-nearest samples.

3. Using the selected STBD, train a PSNN model as SNNcube using unsupervised learning.

4. Train a classifier for the $x 1$ SNN model using deSNN.

During the unsupervised STDP learning process, the neuronal connections are evolved and adapted in the SNNcube. The more spikes transmitted are between two connected neurons at time $t$, the stronger the connection is between them. The SNNcube learns to generate specific trajectories of spiking activities when a particular input pattern is entered. The proposed NeuCube SNN-based personalised modelling framework is graphically shown in Fig.2. The framework is implemented as a PSNN software development system in Matlab that complements the NeuCube SNN generic development system (see www.kedri.aut.ac.nz/neucube/) [18], (Fig.3). 


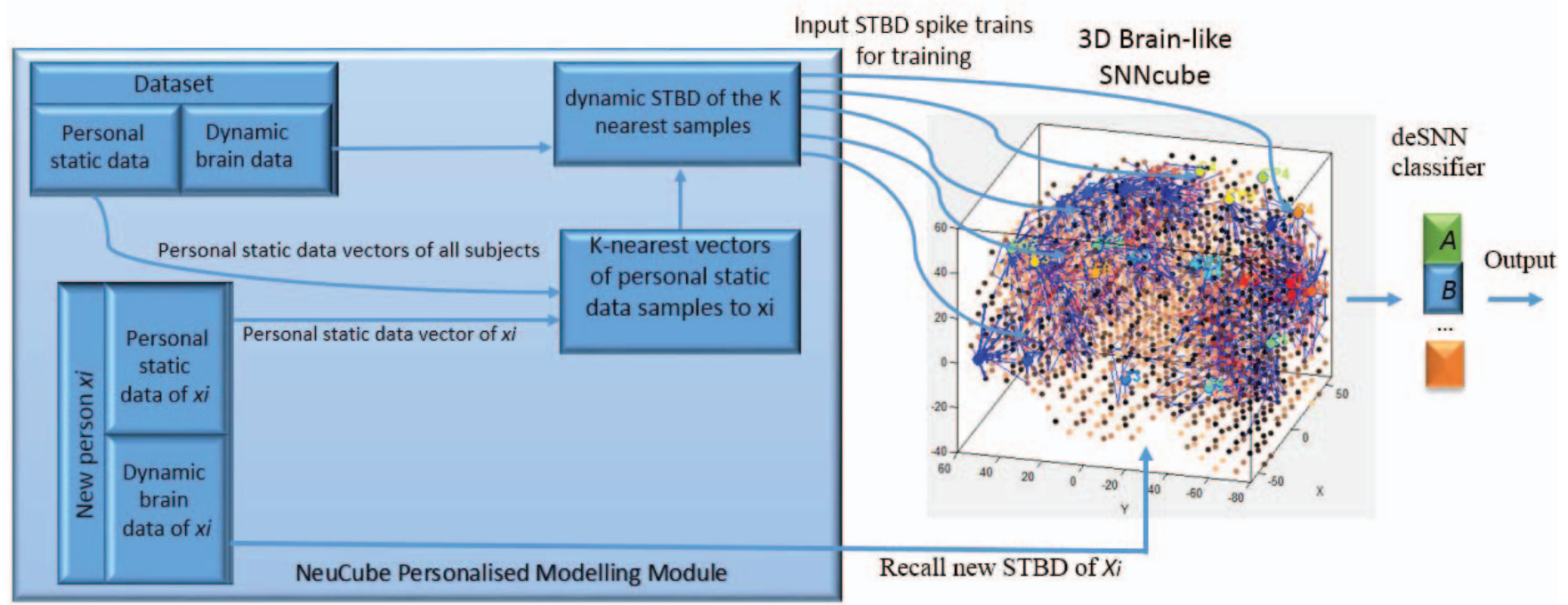

Fig. 2. A block diagram of the NeuCube SNN-based personalised modelling approach. Vector-based personal static data is available, each vector represents a person's static clinical features, such as opiate use duration, methadone dose, etc. For every new input person $x_{i}$, K nearest static data vectors to $x_{i}$ are selected. Then the dynamic STBD of those K nearest subjects are used to train the personalised SNNcube using STDP learning.
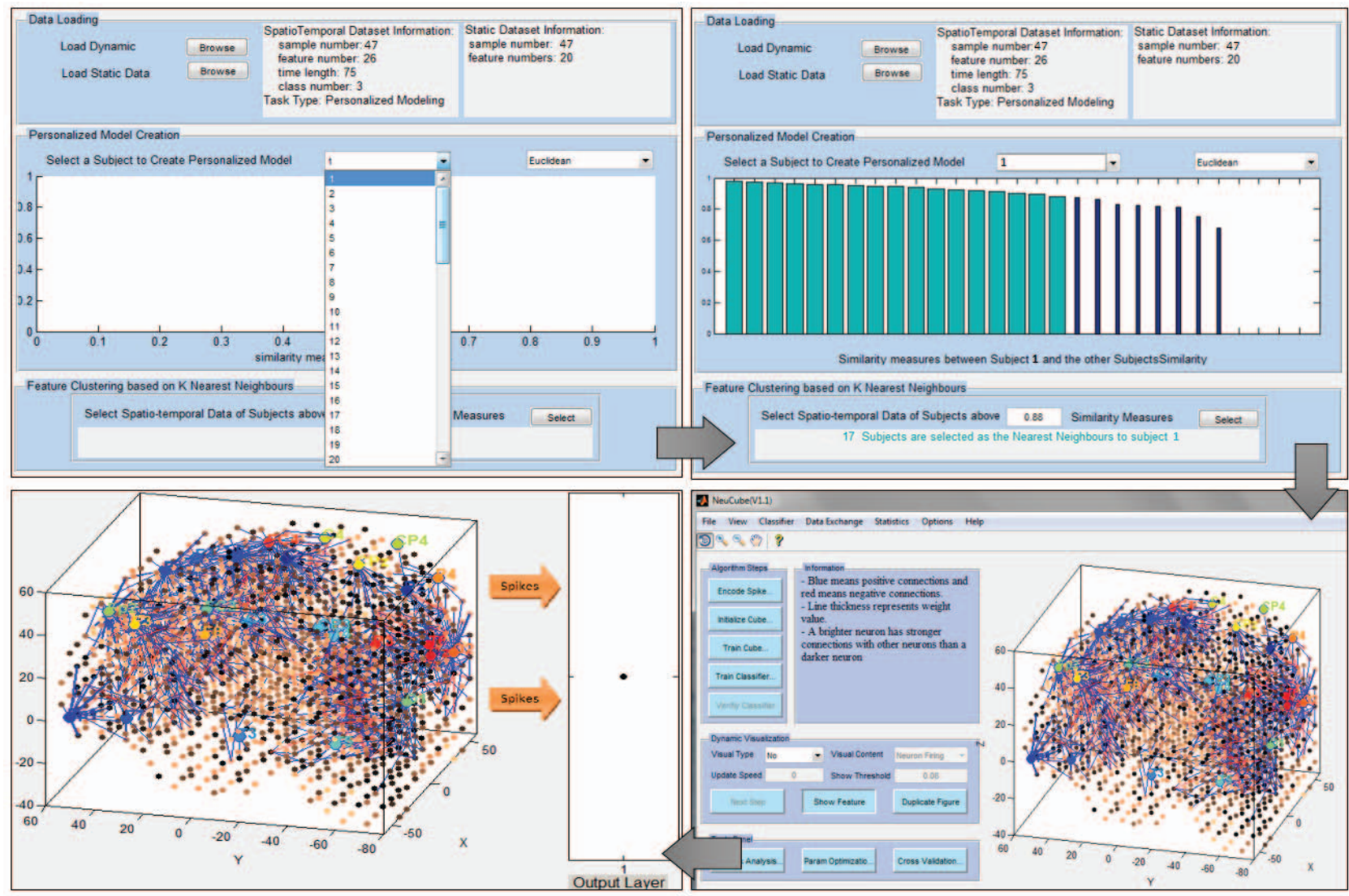

Fig. 3. A NeuCube SNN-based personalised modelling system is developed as part of the NeuCube integrated platform [18]. The EEG STBD and the person static information is loaded; subject ID 1 is selected as an example; a cluster of 17 subjects who have over $88 \%$ similarity to subject 1 is detected. The STBD of these 17 subjects are transferred into the SNNcube for the creation of a personalised model of subject 1. The STBD is encoded into spike trains and mapped into a 3D SNNcube and STDP unsupervised learning is performed. In the output layer, an output neuron is created and connected to all neurons of the SNNcube. Subject 1 STBD is entered to test the classifier. The blue lines are positive (excitatory) connections, while the red lines are negative (inhibitory) connections. The brighter the colour of a neuron, the stronger its activity with neighbouring neurons. Thickness of the lines also identifies the neuron's enhanced connectivity. The 1471 neurons of the brain-like SNNc are spatially located according to the Talairach brain atlas [21] and 26 input neurons are allocated as inputs for the 26 EEG channels 


\section{B. EEG Spatio-Temporal Data Case Study}

To illustrate the proposed NeuCube SNN-based personalised modelling methods and systems for STBD, here we used EEG data collected from two groups of subjects when they performed a cognitive GO- NOGO task. During a GO/NOGO task, a participant is required to perform an action given certain stimuli (e.g. press a button-GO) and inhibit that action under a different set of stimuli (e.g. not press that same button- NOGO).

The collected EEG data consists of 68 samples each representing an EEG STBD of one subject, in which 21 samples are labelled as healthy $(\mathrm{H}), 18$ samples are labelled as opiate addict patients (OP), and 29 samples are labelled as patients undertaking methadone maintenance treatment $(\mathrm{M})$.

The EEG data was recorded via 26 EEG channels: Fp1, Fp2, Fz, F3, F4, F7, F8, Cz, C3, C4, CP3, CPz, CP4, FC3, FCz, FC4, T3, T4, T5, T6, Pz, P3, P4, O1, O2, and Oz.

In addition to the EEG STBD, personal clinical, static information was also recorded per subject, such as: gender, age, opiate use duration, methadone use duration, methadone dose, history of overdose, anger level.

\section{RESULTS}

In this experiment, for every personalised model creation, SNNcube is trained by the most informative EEG data corresponding to subjects with similar clinical static information.

The experiment is illustrated in Figs.3, 4 and Table 1.

The trained PSNN can be used for a better comparatively analysis across individual subject's performance. Fig.3 represents NeuCube SNN-based personalised modelling user interface, which is developed as part of the NeuCube, for creating PSNN models.

Using the proposed framework for PSNN modelling, we created 47 separate personalised SNN models (for 18 OP subjects and $29 \mathrm{M}$ subjects), each trained on a subset of informative EEG data corresponding to a cluster of samples with similar static data. Then the overall obtained accuracy of all 47 SNNcube models is compared with the global SNNcube which was trained on the entire dataset and tested on individual data.

Table I shows that PSNN models results in a better overall classification accuracy when compared with global SNN models using the same NeuCube architecture. Precise interpretation of the person performance can be also obtained as illustrated on 6 subjects in Fig.4.

Fig. 4 shows six PSNN models, each of them created for a single person $x_{i}$. In this figure, the left column represents the similarity between the clinical static data vector of person $x_{i}$ and other subject's static data vectors. The similarity is shown as a bar graph. The green highlighted bar lines represent those subjects that have over $88 \%$ similarity with person $x_{i}$. The EEG data of those subjects are encoded into spike trains and then transferred into a personalised 3D brain-like SNN cube for STDP unsupervised learning. During the learning, the connections between the neurons of the PSNNcube are strengthened or weakened based on the timing of post-synaptic action potential in relation to the pre-synaptic spikes. If presynaptic neuron $i$ spikes first and then post-synaptic neuron $j$ spikes, then the connection weight $W_{i j}$ between these two neurons $i$ and $j$ increases, otherwise it decreases.

As shown in Fig.4, for instance, for M subject id. 1, stronger neuronal connections are evolved around the input EEG channels located in the right hemisphere of the PSNN model corresponding to the right hemisphere. If we compare it with $\mathrm{M}$ subjects id. 2 and id. 3, which reveals a different response to methadone treatment of these 3 subjects, the differences of the model connectivity are observed.

These findings can reveal significant information about the individual person brain functions against a cognitive task and can be further used to suggest a better treatment based on the personalised methadone dose-related effects in case of the experiment presented. This can be used to control individual differences and pre-existing conditions, and help to predict treatment response.

\section{CONCLUSION}

In contrast to the global modelling, personalised modelling creates a specific model for each new person based on existing samples closest to this person's data from a dataset.

In this study, we propose a framework for personalised modelling based on the NeuCube SNN architecture [10].

A NeuCube personalised model includes several methods and algorithms that allow different aspects of EEG data to be studied and analysed: clustering the subjects' STBD based on the K-nearest subject vectors; spatial mapping of the STBD into a 3D personalised SNN structure; unsupervised learning in the SNNcube; visualisation of the connectivity and the spiking activity of the trained SNNcube for the discovery of new information related to the data and the brain processes that generated it; supervised learning in a SNN classifier; and model validation.

Overall, PSNN models trained on a subset of informative EEG STBD resulted in a better classification accuracy when compared with global SNN models. In addition, they can be used to reveal individual characteristics on brain activities that can be used to find the best patient- oriented treatment.

TABLE I. Classification accuracy obtained via NeuCube personalised modelling vs. using a global classification NeuCube model (reported in our previous study [3]). Each PSNNcube is trained by STBD of subjects with similar static data and then tested by a new person's STBD.

\begin{tabular}{|c|c|c|}
\hline Methods & NeuCube-Personalised modelling & NeuCube- Global modelling \\
\hline $\begin{array}{c}\text { Classification accuracy of } \\
\text { class M versus class OP in \% }\end{array}$ & Averaged over 47 trained PSNNcubes: & $\begin{array}{c}\text { One trained SNNcube using } \\
\text { all subjects and tested via } \\
\text { leave-one-out method: 79.00 }\end{array}$ \\
\hline
\end{tabular}




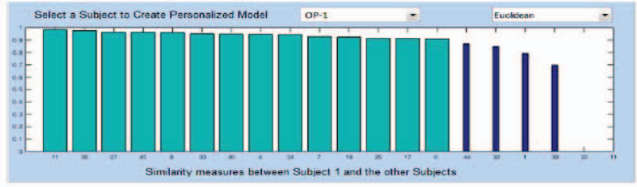

OP subject id:1

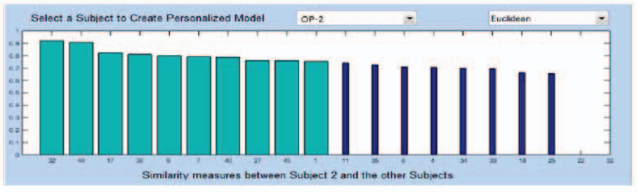

OP subject id:2

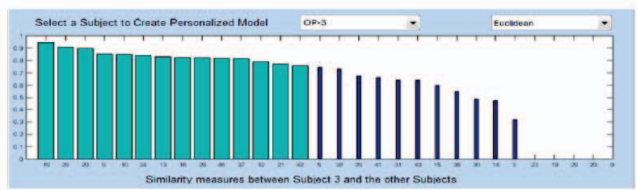

OP subject id:3

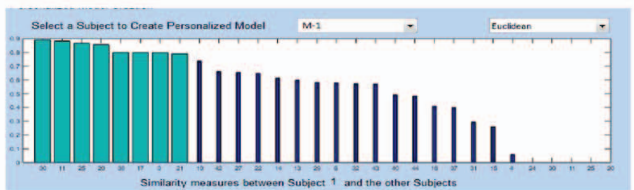

M subject id:1

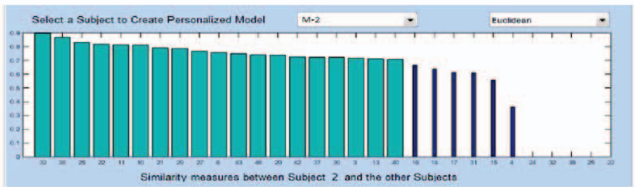

M subject id: 2
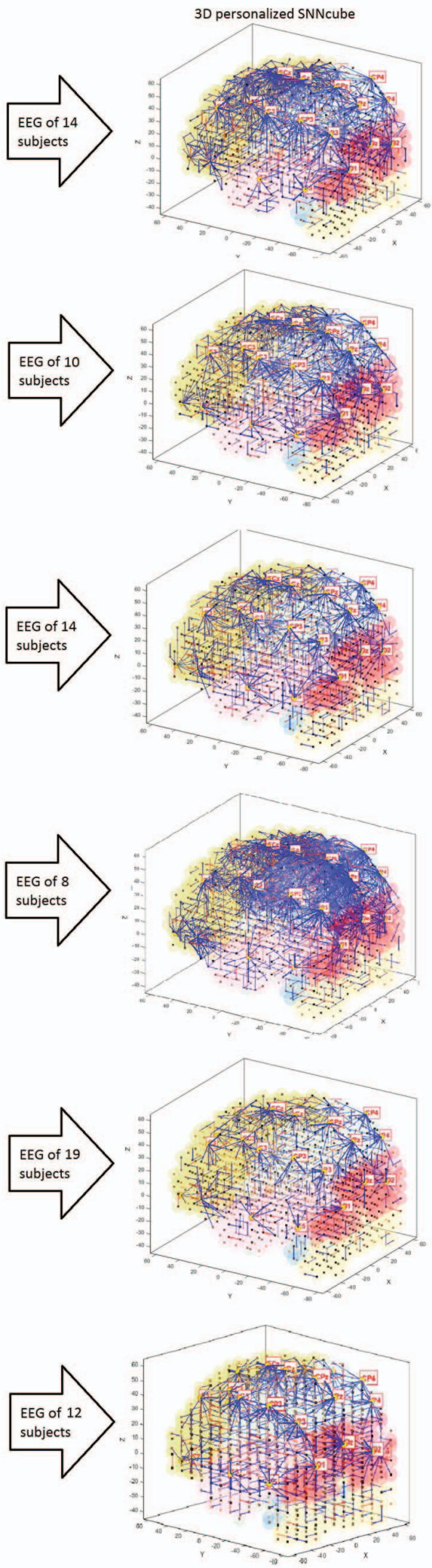

$2 D$ representation (projection) of the NNcube
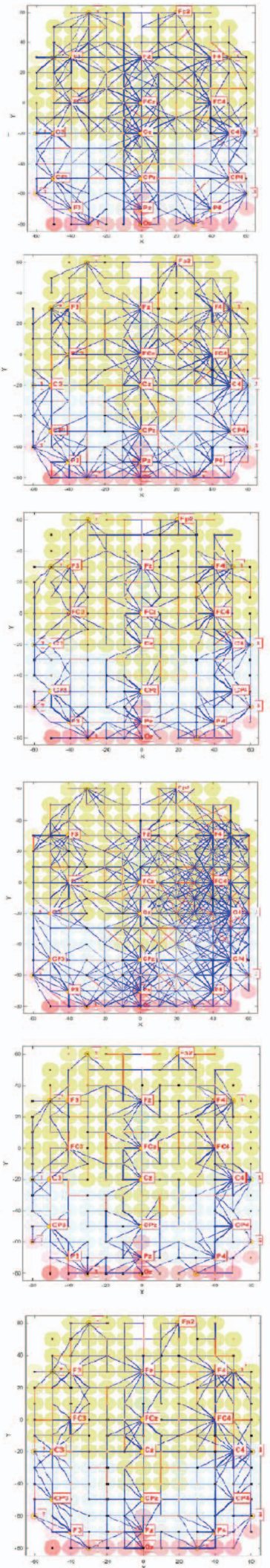

Fig. 4. The differences between 6 randomly subjects in relation to their static, clinical data and brain activities achieved through building personalised models in NeuCube. The 1471 neurons of the brain-like SNNc are spatially located according to the Talairach brain atlas [21] and 26 input neurons are allocated as inputs for the 26 EEG channels. For every individual, one PSNNcube is trained by a subset of EEG data corresponding to the subjects with similar static data. 


\section{ACKNOWLEDGMENT}

The research is supported by the Knowledge Engineering and Discovery Research Institute of the Auckland University of Technology (www.kedri.aut.ac.nz), New Zealand. The authors would like to acknowledge Professor Robert Kydd, Dr Bruce Russell, and Dr Grace Wang for providing us with the EEG data. A limited version of the NeuCube SNN development system along with a benchmark EEG data are available at http://www.kedri.aut.ac.nz/neucube .

\section{REFERENCES}

[1] E. Niedermeyer and F. L. da Silva, Electroencephalography: basic principles, clinical applications, and related fields, 5, Ed., Lippincott Williams \& Wilkins, 2005.

[2] S. Ogawa, D. W. Tank, R. Menon, J. M. Ellermann, S. G. Kim, H. Merkle and K. gurbil, "Intrinsic signal changes accompanying sensory stimulation: functional brain mapping with magnetic resonance imaging," Proceedings of the National Academy of Sciences, vol. 89, no. 13, pp. 5951-5955, 1992.

[3] M. Gholami Doborjeh, G. Wang, N. Kasabov, R. Kydd and B. R. Russell, A Spiking Neural Network Methodology and System for Learning and Comparative Analysis of EEG Data from Healthy versus Addiction Treated versus Addiction Not Treated Subjects, IEEE Tr. BME, doi:10.1109/TBME.2015.2503400, 2015

[4] N. Kasabov and Y. Hu, "Integrated optimisation method for personalised modelling and case studies for medical decision support," International Journal of Functional Informatics and Personalised Medicine, vol. 3, no. 3, pp. 236-256, 2010.

[5] W. Maass, N. Thomas and M. Henry, "Real-time computing without stable states: a new framework for neural computation based on perturbations," Neural Computation, vol. 14, no. 11, p. 2531-2560, 2002.

[6] S. M. Bohte and J. N. Kok, "Applications of spiking neural networks," Information Processing Letters, vol. 95, no. 6, pp. 519-520, 2005.

[7] R. Brette, M. Rudolph, T. Carnevale, M. Hines, D. Beeman and J. Bower, "Simulation of networks of spiking neurons: a review of tools and strategies," Journal of computational neuroscience, vol. 23, no. 3, pp. 349-398, 2007.

[8] N. Kasabov, K. Dhoble, N. Nuntalid and G. Indiveri, "Dynamic evolving spiking neural networks for on-line spatio-and spectro-temporal pattern recognition," Neural Networks, vol. 41, pp. 188-201, 2013.

[9] N.Kasabov, Z. HOU, V. FEIGIN, Y. CHEN, "Improved method and system for predicting outcomes based on spatio / spectro-temporal data", PCT patent, WO 2015030606 A2, 2015.
[10] N. Kasabov, "NeuCube: A spiking neural network architecture for mapping, learning and understanding of spatio-temporal brain data," Neural Networks, vol. 52, pp. 62-76, 2014.

[11] "EU Human Braib Project (HBP)," [Online]. Available: www.thehumanbrainproject.eu.

[12] "USA Brain Initiative," [Online]. Available: http://www.nih.gov/science/brain/.

[13] S. Furber, D. R. Lester, L. Plana and J. D. Garside, "Overview of the spinnaker system architecture," IEEE Transactions on Computers, vol. 62, no. 12, pp. 24542467, 2013.

[14] S. Furber, "To build a brain," IEEE Spectrum, vol. 49, no. 44-49, pp. 44-49, 2012.

[15] G. Indiveri and T. K. Horiuchi, "Frontiers in neuromorphic engineering," Frontiers in neuroscience, vol. 5, 2011.

[16] G. Indiveri, B. Linares-Barranco, T. J. Hamilton and A. Van Schaik, "Neuromorphic silicon neuron circuits," Frontiers in neuroscience, vol. 5, 2011.

[17] G. Indiveri, E. Chicca and R. J. Douglas, "Artificial cognitive systems: from VLSI networks of spiking neurons to neuromorphic cognition.," Cognitive Computatio, vol. 1, no. 2, pp. 119-127, 2009.

[18] N. Kasabov et al, "Design methodology and selected applications of evolving spatio- temporal data machines in the NeuCube neuromorphic framework," Neural Networks, accepted and on-line published 2015, 2016

[19] N. Kasabov, "Evolving connectionist systems for adaptive learning and knowledge discovery: Trends and Directions," Knowledge Based Systems, 2015, http://dx.doi.org/10.1016/j.knosys.2014.12.032.

[20] N. Kasabov, V. Feigin, Z. G. Hou, Y. Chen, L. Liang, R. Krishnamurthi, P. Parmar, "Evolving spiking neural networks for personalised modelling, classification and prediction of spatio-temporal patterns with a case study on stroke," Neurocomputing, no. 134, pp.269-279. doi:10.1016/j.neucom.2013.09.049, 2014

[21] L. Koessler, L. Maillard, A. Benhadid, J. P. Vignal, J. Felblinger, H. Vespignani and M. Braun, "Automated cortical projection of EEG sensors: anatomical correlation via the international 10-10 system," Neuroimage, vol. 46, no. 1, pp. 64-72, 2009.

[22] T. Masquelier, R. Guyonneau and S. J. Thorpe, "Competitive STDP-based spike pattern learning," Neural computation, vol. 21, no. 5, pp. 1259-1276, 2009.

[23] E. Tu, N. Kasabov, J. Yang "Mapping Temporal Variables into the NeuCube for Improved Pattern Recognition, Predictive Modelling and Understanding of Stream Data," IEEE Transactions on Neural Networks and Learning Systems, doi:10.1109/TNNLS.2016.2536742, 2016. 